\title{
Erratum to: Temozolomide and 13-cis retinoic acid in patients with anaplastic gliomas: a prospective single-arm monocentric phase-II study (RNOP-05)
}

\author{
Oliver Grauer - Christina Pascher - Christian Hartmann - Florian Zeman • \\ Michael Weller • Martin Proescholdt • Alexander Brawanski - Thorsten Pietsch • \\ Wolfgang Wick $\cdot$ Ulrich Bogdahn $\cdot$ Peter Hau
}

Published online: 20 October 2011

(C) Springer Science+Business Media, LLC. 2011

Erratum to: J Neurooncol (2011) 104:801-809

DOI:10.1007/s11060-011-0548-y

There is an error in Table 4 of the original publication. The entry for the status of $I D H I$ indicated as 'Not Mutated' should read 'Mutated'.

The online version of the original article can be found under doi:10.1007/s11060-011-0548-y.

O. Grauer · C. Pascher $\cdot$ U. Bogdahn $\cdot$ P. Hau $(\bowtie)$

Department of Neurology, University Medical Center

Regensburg, Universitätsstrasse 84, 93053 Regensburg,

Germany

e-mail: peter.hau@medbo.de

C. Hartmann

Clinical Cooperation Unit Neuropathology (G380), German

Cancer Research Center, Heidelberg, Germany

C. Hartmann

Department of Neuropathology, Institute of Pathology,

University of Heidelberg, Im Neuenheimer Feld 220,

69120 Heidelberg, Germany

F. Zeman

Center for Clinical Studies, University Medical Center

Regensburg, Franz-Josef-Strauß-Allee 11, 93053 Regensburg,

Germany
M. Weller

Department of Neurology, University Hospital Zürich,

Frauenklinikstrasse 26, 8091 Zurich, Switzerland

M. Proescholdt $\cdot$ A. Brawanski

Department of Neurosurgery, University Medical Center

Regensburg, Franz-Josef-Strauß Allee 11, 93053 Regensburg,

Germany

T. Pietsch

Department of Neuropathology, University of Bonn,

Sigmund-Freud-Str. 3, 53015 Bonn, Germany

W. Wick

Department of Neurooncology, Neurology Clinic and National Center for Tumor Disease, University of Heidelberg,

Im Neuenheimer Feld 400, 69120 Heidelberg, Germany 